\title{
BOUNDARY VALUE PROBLEMS FOR DEGENERATE ELLIPTIC-PARABOLIC EQUATIONS OF THE FOURTH ORDER
}

\author{
ROBERT G. ROOT
}

\begin{abstract}
We consider boundary value problems for the fourth-order linear equation

$$
A^{i j r s} u_{i j r s}+A^{i j r} u_{i j r}+A^{i j} u_{i j}-\gamma\left(a^{i j} u_{i}\right)_{j}+A^{i} u_{i}+F u=f \text { in } \bar{\Omega}
$$

with smooth coefficients. The fourth-order part may degenerate on arbitrary subsets of $\bar{\Omega}$, i.e., $A^{i j r s}(x) m_{i j} m_{r s} \geq 0$ for all $n \times n$ matrices $M$, with no restriction on where equality occurs. We assume the $a^{i j}$ part of the operator is uniformly elliptic (of second order) on $\Omega$ while $\gamma$ is a parameter allowing us to increase modulus of ellipticity as much as needed. As in Fichera's second-order elliptic-parabolic equations [see, for example, Sulle equazioni differenziali lineari ellitico-paraboliche del secondo ordine, Atti Accad. Naz. Lincei Mem. (8) 5 (1956), 1-30], because of the degeneracy, there may be characteristic portions of the boundary; however, we restrict our attention to the noncharacteristic case. We define a weak solution to the Dirichlet problem and obtain existence and uniqueness results. The question of regularity is addressed; elliptic regularization is used to obtain a Sobolev-type global regularity result. The equation models an anisotropic, inhomogeneous plate under tension that can lose stiffness at any point and in any direction. The regularity result has the satisfying physical interpretation that sufficient tension results in a smooth solution.
\end{abstract}

\section{INTRODUCTION}

In this paper we consider a class of degenerate elliptic linear operators of fourth order that are an analog of Fichera's elliptic-parabolic operators of the second order. General degeneracy is permitted anywhere in the interior of the domain under consideration, but the nature of the degeneracy is restricted at the boundary. The class includes a model of an elastic plate that loses stiffness. The main results are existence, uniqueness, and regularity theorems for weak solutions of appropriate homogeneous first (Dirichlet) boundary value problems.

Received by the editors February 24, 1989.

1980 Mathematics Subject Classification (1985 Revision). Primary 35J70, 35J40, 73C30; Secondary $35 \mathrm{G} 05,73 \mathrm{C} 40$.

Key words and phrases. Elliptic-parabolic, degenerate elliptic, 4th order higher order, elastic plate, anisotropic, inhomogeneous, plate under tension.

Some of the results of this paper were presented at the AMS/MAA Annual Meeting in Atlanta, Georgia, January 1988; the remainder at the Southeast-Atlantic Conference on Differential Equations in Athens, Georgia, November 1988. 
In the 1950's, Fichera initiated a theory of elliptic-parabolic operators of the second order where the degeneracies are arbitrary (see, for example, [ 5 and 6]). Fichera's foundational contributions included the weak formulation for the problem and the existence of a weak solution. He also proved a maximum principle, which led to uniqueness results for classical solutions. The very difficult question of uniqueness for weak solutions was settled by Oleinik [16], who also laid the foundations for the regularity theory in the second-order case [14, 15]. It was Kohn and Nirenberg who, in [11] and [12], provided the Sobolev regularity theorems that inspired the work presented in this article. Subsequently, Phillips and Sarason, in addition to contributing significantly to the regularity theory, generalized the concept of elliptic-parabolic equations to symmetric systems of equations in [19 and 20]. Tartakoff [23] and Bertiger and Cosner [1] also obtained results concerning systems of second-order elliptic-parabolic equations. Tartakoff's results are especially worthy of mention here since he obtained regularity results in the absence of uniqueness for weak solutions, as we do. In 1971, Oleinik and Radkevic [18] compiled a compendium of results for the field that still serves as a standard reference.

The extension of the concepts and tools of the second-order elliptic-parabolic operators to fourth-order operators is largely the work of Weinacht. He has formulated well-posed weak versions of boundary value problems for two types of selfadjoint fourth-order equations: those arising from iterating second-order elliptic-parabolic operators [25], and degenerate fourth-order operators that are selfadjoint but not of iterated form (double-divergence operators) [26]. He has also contributed some preliminary results in the field of eigenvalue asymptotics for these operators, and has obtained regularity results [27] for the case of iterates of second-order elliptic-parabolic operators. Eposito has also worked with fourth-order elliptic-parabolic equations in [4] where he obtains regularity results comparable to those presented here for a special case of our operator. He also claims to treat the case of a characteristic boundary in a forthcoming paper.

The equation studied here can arise as a strongly elliptic operator in a continuum mechanical model of an inhomogeneous, anisotropic elastic plate under tension; the boundary conditions correspond to clamping the plate at the edges. The derivation of this model in the hyperelastic case is an obvious adaptation of the model for the usual homogeneous, isotropic case, as, for example, that presented by Timoshenko and Woinowsky-Krieger [24] or Duvaut and Lions [3] (in Chen [2], the anisotropic but homogeneous case is derived in just this way). The resulting equation has a selfadjoint double-divergence operator, but the operator considered in this paper need not be selfadjoint, and hence the results below apply to plate models that do not assume hyperelasticity. The degeneracy assumed here is equivalent to allowing the plate to lose stiffness either entirely or in just one direction (like a fabric with a stiff warp but a pliant weft). The total or partial loss of stiffness can occur in any subregion (subject to certain smoothness conditions). In terms of elasticity, the regularity results 
given in this exposition show that within the linear model and under the given constraints, sufficient tension guarantees a smooth solution. Greenlee [10] has studied the eigenvalue problem for the related case of a plate-membrane system.

The techniques used in this paper seem to extend to an "elliptic-parabolic" operator of $2 m$ th order in a straightforward fashion. The $m$ th order quadratic form (the characteristic form) for this class of equations would be nonnegative with a uniformly elliptic $(2 m-2)$ th order part (or $(m-1)$ th order quadratic form) to compensate for the degeneracy. The three theorems given in this paper have clear analogs for this class of operators, and their proofs would be close to those given here. The operator treated here has a sort of Gårding's inequality (see the footnote in $\S 3$ ) that will allow a weakened Fredholm alternative (weakened because of the difficulty in obtaining uniqueness of weak solutions, and the coincident restriction on when the Gårding's inequality holds). This paper forgoes the interesting questions of the nature of this operator's spectrum, pursuing instead conditions that guarantee a well-posed boundary value problem. The interested reader may find out more about fourth-order elliptic-parabolic equations, and in particular the logical extensions of this work to operators that allow complete degeneracy at the boundary (in fact, in entire neighborhoods of the boundary), or different boundary value problems from [21, 22, and 27].

\section{THE BOUNDARY VALUE PROBLEM AND ITS WEAK FORMULATION}

We consider the equation (using here and in the following - unless specifically mentioned otherwise-summation notation):

$$
L[u]:=A^{i j r s} u_{i j r s}+A^{i j r} u_{i j r}+A^{i j} u_{i j}+A^{i} u_{i}+F u-\gamma\left(a^{i j} u_{i}\right)_{j}=f \text { in } \Omega
$$

where $\Omega$ is a bounded region in $\mathbf{R}^{n}$ with smooth boundary. The degenerate nature of the operator $L$ is given by the following condition:

$$
A^{i j r s}(x) m_{i j} m_{r s} \geq 0 \quad \forall \mathbf{M} n \times n \text { matrices, at any } x \in \bar{\Omega} .
$$

If the inequality were strict for symmetric $\mathbf{M} \neq \mathbf{0}$, it follows that

$$
A^{i j r s}(x) v_{i} v_{j} v_{r} v_{s}>0 \quad \forall \text { nonzero } \mathbf{v} \in \mathbf{R}^{n}, \forall x \in \bar{\Omega}
$$

i.e., the operator would be strongly elliptic; our operator may degenerate from ellipticity, and is therefore called elliptic-parabolic. We place the following restriction on the degeneracy. At any point on the boundary of the region of interest, we represent the unit outer normal to $\partial \Omega$ by $\mathbf{n}$. At such a point, it must be that

$$
A^{i j r s} n_{i} n_{j} n_{1} n_{s}>0 \text {. }
$$

Portions of the boundary where this requirement fails are called characteristic, and the boundary conditions stated below do not give a well-posed problem if there is a characteristic portion of the boundary (see [21 or 26]).

In order to compensate for the degeneracy of the highest-order part of the operator, we will require that the second-order part of the operator be uniformly 
elliptic and that its modulus of ellipticity be as large as we need. This is accomplished by including the term $\gamma\left(a^{i j} u_{i}\right)_{j}$. We will assume without loss of generality that the modulus of ellipticity for this expression is $\gamma$, that is,

$$
a^{i j} \xi^{i} \xi^{j} \geq|\boldsymbol{\xi}|^{2} \quad \forall \xi \in \mathbf{R}^{n}, \forall x \in \bar{\Omega} .
$$

Thus, the parameter $\gamma$ allows us to make the modulus of ellipticity for the entire second-order part of the operator as large as we choose, and we will use this ability in all the proofs that follow. It is assumed that the other coefficients of the operator, in particular $F$ (in contrast with the analogous system in Tartakoff's work), are independent of $\gamma$; however, the nature and importance of the dependence on $\gamma$ is discussed briefly in the remark after the proof of Theorem 1 in the next section.

The boundary conditions for the Dirichlet problem for our operator as described above differ not at all from those of the classical fourth-order elliptic Dirichlet problem; the homogeneous problem involves specifying

$$
u=0 \text { and } \frac{\partial u}{\partial n}=0 \quad \text { on } \partial \Omega .
$$

We now show that this problem has a well-posed weak formulation in a suitably chosen function space.

To obtain this weak formulation, the coefficients $A^{i j r s}, A^{i j r}$, and $A^{i j}$ are all assumed to have the following symmetry of their indices:

$$
A^{i j r s}=A^{j i r s}=A^{r s i j}, \quad A^{i j r}=A^{j i r}=A^{i r j}, \quad A^{i j}=A^{j i}, \quad \text { and } \quad a^{i j}=a^{j i},
$$

as well as the other relations implied by these. Notice that we do not assume complete interchangeability of indices for the fourth-order coefficients; this allows certain elliptic operators to be included in this class. Consider, for instance, the two-dimensional uniformly elliptic operator:

$$
u_{x x x x}+u_{y y y y}-u_{x x y y} .
$$

We would like this to qualify as the principal part of an elliptic-parabolic operator, but to do this we specify the coefficients in the following way:

$$
A^{1111}=A^{2222}=1, \quad A^{1122}=A^{2211}=-1 / 2, \quad 0=A^{1212}=A^{2112}=\text { etc. },
$$

rather than specifying that the six coefficients whose indices contain two 1's and two 2's each be $-1 / 6$. Thus, this deviation from complete symmetry permits the broadest class of principal parts to the operator, including some uniformly elliptic operators that would be excluded under more stringent symmetry conditions. (It is easy to verify that any positive semidefinite quadratic form on symmetric matrices has a representation as a fourth-order tensor satisfying the symmetry conditions on the fourth-order coefficients given in [5].) We also assume that the coefficients satisfy the following smoothness conditions:

$$
A^{i j r s} \in C^{3}(\bar{\Omega}) ; \quad A^{i j r} \in C^{2}(\bar{\Omega}) ; \quad A^{i j}, a^{i j} \in C^{1}(\bar{\Omega}) ; \quad A^{i}, F \in C^{0}(\bar{\Omega}) .
$$


Under these assumptions, we apply the operator $L$ to any $u \in H^{4}(\Omega)$ and take the $L^{2}(\Omega)$ inner product with a function $v$ from $H_{0}^{2}(\Omega)$. After several applications of the divergence theorem, we arrive at the following:

$$
\begin{aligned}
(L[u], v)= & \left(A^{i j r s} u_{i j}, v_{r s}\right)+\left(\left[-\left(A_{s}^{i j r s}+A_{s}^{i s j r}\right)+A^{i j r}\right] u_{i}, v_{j r}\right) \\
& +\gamma\left(a^{i j} u_{i}, v_{j}\right)+\left(\left[-3 A_{r s}^{i r j s}+2 A_{r}^{i j r}-A^{i j}\right] u_{i}, v_{j}\right) \\
& +\left(\left[-A_{j r s}^{i j r s}+A_{j r}^{i j r}-A_{j}^{i j}+A^{i}\right] u_{i}, v\right)+(F u, v),
\end{aligned}
$$

where we have used $(\cdot, \cdot)$ to indicate the $L^{2}(\Omega)$ inner product. We rename the coefficients above by

$$
\begin{aligned}
B^{i j r} & :=-\left(A_{s}^{i j r s}+A_{s}^{i s j r}\right)+A^{i j r}, \\
C^{i j} & :=-3 A_{r s}^{i r j s}+2 A_{r}^{i j r}-A^{i j}, \\
E^{i} & :=-A_{j r s}^{i j s}+A_{j r}^{i j r}-A_{j}^{i j}+A^{i},
\end{aligned}
$$

and define the bilinear form associated with the operator $L$ by

$$
\begin{aligned}
B(u, v):= & \left(A^{i j r s} u_{i j}, v_{r s}\right)+\left(B^{i j r} u_{i}, v_{j r}\right)+\gamma\left(a^{i j} u_{i}, v_{j}\right) \\
& +\left(C^{i j} u_{i}, v_{j}\right)+\left(E^{i} u_{i}, v\right)+(F u, v) .
\end{aligned}
$$

In deriving the bilinear form, we assumed that the second argument belonged to $H_{0}^{2}(\Omega)$, which implies that the boundary conditions (4) are satisfied, in the sense of trace. Note that the operator $L$ can, under the stipulated smoothness assumptions, be written in divergence form as follows:

$$
L[u]=\left(A^{i j r s} u_{i j}\right)_{r s}+\left(B^{i j r} u_{i}\right)_{j r}-\gamma\left(a^{i j} u_{i}\right)_{j}-\left(C^{i j} u_{i}\right)_{j}+E^{i} u_{i}+F u .
$$

Define the inner product

$$
(u, v)_{\mathscr{R}}:=\left(A^{i j r s} u_{i j}, v_{r s}\right)+\int_{\Omega} \nabla u \cdot \nabla v d x,
$$

and take the completion of $C_{0}^{\infty}(\Omega)$ under the associated norm to define the Hilbert space $\mathscr{H}$, in which our weak solutions will lie. Note that the set $\mathscr{H}$ is a linear manifold in $H_{0}^{1}(\Omega)$. We are now in a position to give our weak formulation of the boundary value problem.

Definition. A function $u \in \mathscr{H}$ will be called an $\mathscr{H}$-weak solution of the homogeneous Dirichlet problem (1), (4), if

$$
B(u, v)=(f, v) \quad \forall v \in C_{0}^{\infty}(\Omega) .
$$

As a first step toward the proofs of the theorems given in the next section, we now show that for every $v \in H_{0}^{2}(\Omega), B(\cdot, v)$ is a bounded linear functional on $\mathscr{H}$. To see this, let $u \in \mathscr{H}$ be chosen arbitrarily. By the definition of the degeneracy (2) and the symmetry relations in (5), the $A^{i j r s}$ term of the bilinear form is symmetric and positive semidefinite, and therefore a Schwarz inequality obtains. Applying this Schwarz inequality to the first term of the bilinear form 
and the $L^{2}$ Schwarz inequality to the other terms, we arrive after some simple bounding at the expression

(6)

$$
\begin{aligned}
|B(u, v)| \leq & {\left[\left(A^{i j r s} u_{i j}, u_{r s}\right)^{1 / 2}+\left(\int_{\Omega}|\nabla u|^{2} d x\right)^{1 / 2}+\|u\|_{0}\right] } \\
& \times\left[\left(A^{i j r s} v_{i j}, v_{r s}\right)^{1 / 2}+\left(\int_{\Omega}\left|\sum_{i=1}^{n} B^{i j r} v_{j r}\right|^{2} d x\right)^{1 / 2}\right. \\
& +\left(\int_{\Omega}\left|\sum_{i=1}^{n} C^{i j} v_{j}\right|^{2} d x\right)^{1 / 2}+\gamma\left(\int_{\Omega}\left|\sum_{i=1}^{n} a^{i j} v_{j}\right|^{2} d x\right)^{1 / 2} \\
& \left.+\left(\int_{\Omega}\left|\sum_{i=1}^{n} E^{j} v\right|^{2} d x\right)^{1 / 2}+\left(\int_{\Omega}(F v)^{2} d x\right)^{1 / 2}\right],
\end{aligned}
$$

where $\|\cdot\|_{0}$ represents the norm of $L^{2}(\Omega)$. Since $u$ must be in $H_{0}^{1}(\Omega)$, we can apply the Poincaré-Friedrichs inequality to the first factor above to arrive at the bound:

$$
|B(u, v)| \leq C\|v\|_{2}\|u\|_{\mathscr{H}}
$$

where the constant $C$ depends only on the region $\Omega$ and the coefficients of the operator $L$, and $\|\cdot\|_{2}$ represents the $H^{2}(\Omega)$ norm. So, in fact, we have shown that the bilinear form is bicontinuous on $\mathscr{H} \times H^{2}(\Omega)$. Thus, a statement equivalent to the preceding definition would be: $\mathscr{H}$-weak solutions solve $B(u, v)=(f, v) \quad \forall v \in H_{0}^{2}(\Omega)$, since $C_{0}^{\infty}(\Omega)$ is dense in $H_{0}^{2}(\Omega)$.

\section{THE WELL-POSEDNESS OF THE WEAK FORMULATION}

In this section, we present the existence and uniqueness results available for the boundary value problem formulated in the preceding section. Our existence result also provides us with uniqueness of sufficiently smooth solutions.

Theorem 1. There exists a weak solution to the boundary value problem (1), (4), as defined in the previous section, provided the parameter $\gamma$ is sufficiently large. Further, for $\gamma$ sufficiently large, any $\mathscr{H}$-weak solution to the boundary value problem (1), (4) in $H_{0}^{2}(\Omega)$ is unique in $H_{0}^{2}(\Omega)$.

The proof given below is based on that of Oleinik for the case of second-order elliptic-parabolic operators in [19]. This proof in turn seems to be a restatement of that of Fichera [6].

Proof. We have shown that $B(\cdot, v)$ is a continuous linear functional on the space $\mathscr{H}$ for any $v \in H_{0}^{2}(\Omega)$. Thus the Riesz Representation Theorem assures us that there is an element $T v \in \mathscr{H}$ such that $B(u, v)=(u, T v)_{\mathscr{H}}$ for all $u \in \mathscr{H}$. Clearly $T$ is a linear operator, but the crux of our proof is showing 
that $T$ has a bounded inverse on the closure of its range, which we will denote as:

$$
{\overline{T\left(H_{0}^{2}(\Omega)\right)}}^{\mathscr{H}}=: \mathscr{H}^{\prime} .
$$

To obtain this result, it is sufficient to show that there exists a $\rho>0$ such that $\|T v\|_{\mathscr{L}} \geq \rho\|v\|_{\mathscr{H}}$, for from this it follows that (i) $T$ is one to one and therefore the inverse, $T^{-1}$, is defined on the range, and (ii) the inverse is bounded with norm $1 / \rho$. From this the extension of $T^{-1}$ to all of $\mathscr{H}^{\prime}$ follows easily (see, e.g., Goldberg [9, Theorem II.2.1]).

Notice that for $v \in H_{0}^{2}(\Omega)$, we can apply the divergence theorem and the symmetry of the coefficients given in (5) to see that

$$
\left(B^{i j r} v_{i}, v_{j r}\right)=-\frac{1}{2}\left(B_{r}^{i j r} v_{i}, v_{j}\right) \text {. }
$$

Thus, by applying this identity and the Schwarz and arithmetic-geometric mean inequalities to the first-order term, we have that

$$
\begin{aligned}
B(v, v) \geq & \left(A^{i j r s} v_{i j}, v_{r s}\right)+\int_{\Omega}\left[\gamma a^{i j}+C^{i j}-\frac{1}{2} B_{r}^{i j r}-\left(E^{i}\right)^{2} \delta_{j}^{i}\right] v_{i} v_{j} d x \\
& -\int_{\Omega} v^{2} d x+\int_{\Omega} F v^{2} d x
\end{aligned}
$$

where $\delta_{j}^{i}$ is a Kroenecker delta. Notice now that the modulus of ellipticity associated with the second-order part will be a monotone-increasing function of $\gamma$. Thus, for any $\tau>0$, there is a choice of the parameter $\gamma$ that results in

$$
\left[\gamma a^{i j}+C^{i j}-\frac{1}{2} B_{r}^{i j r}-\left(E^{i}\right)^{2} \delta_{j}^{i}\right] \xi^{i} \xi^{j} \geq \tau|\boldsymbol{\xi}|^{2} \quad \forall \boldsymbol{\xi} \in \mathbf{R}^{n}
$$

for fixed $\gamma$, we will call the maximum $\tau$ for which this inequality holds $\tau(\gamma)$. Define $t:=\inf _{x \in \Omega} F(x)-1$; then for any $\gamma$ such that $\tau(\gamma)$ is positive, we have that

$$
B(v, v) \geq\left(A^{i j r s} v_{i j}, v_{r s}\right)+\tau \int_{\Omega}|\nabla v|^{2} d x+t\|v\|_{0}^{2}
$$

(see note below ${ }^{1}$ ) and so, if $t$ is nonnegative, it follows that

$$
B(v, v) \geq \min \{1, \tau\}\|v\|_{\mathscr{L}}^{2} \text {. }
$$

If, on the other hand, $t$ is negative, then expressing the Poincaré-Friedrichs inequality as

$$
\int_{\Omega}|\nabla v|^{2} d x \geq \lambda \int_{\Omega} v^{2} d x \quad \forall v \in H_{0}^{1}(\Omega),
$$

we can stipulate that $\gamma$ be chosen sufficiently large so that $\tau(\gamma)>-t / \lambda>0$. Call $\tau^{\prime}:=\tau+t / \lambda$, and we see that

$$
B(v, v) \geq \min \left\{1, \tau^{\prime}\right\}\|v\|_{\mathscr{H}}^{2} .
$$

\footnotetext{
${ }^{1}$ This is the Gårding's inequality mentioned in the introduction. Note that it holds only for functions belonging to $H_{0}^{2}(\Omega)$. Thus we can obtain a Fredholm alternative with uniqueness only for solutions at least this smooth.
} 
Call the appropriate "min" $\rho$, and the desired estimate follows from

$$
\rho\|v\|_{\mathscr{H}}^{2} \leq B(v, v)=(v, T v)_{\mathscr{H}} \leq\|v\|_{\mathscr{H}}\|T v\|_{\mathscr{H}} .
$$

To complete the existence proof, first note that

$$
\left|\int_{\Omega} f v d x\right| \leq\|f\|_{0}\|v\|_{0} \leq\|f\|_{0} \frac{1}{\lambda}\|v\|_{\mathscr{H}} \leq \frac{\|f\|_{0}}{\rho \lambda}\|T v\|_{\mathscr{Z}},
$$

where we have used the Poincaré-Friedrichs inequality and the second term of the $\mathscr{H}$ norm to bound the $L^{2}$ norm of $v$. Thus, the $L^{2}$ inner product with $f$ defines a continuous linear functional on $\left\{T v: v \in H_{0}^{2}(\Omega)\right\}$, and therefore on all of $\mathscr{H}^{\prime}$. So, by the Riesz Representation Theorem, there exists a unique $u \in \mathscr{H}^{\prime}$ such that $(f, v)=(u, T v)_{\mathscr{H}}=B(u, v)$. Of course, $u$ is not unique in $\mathscr{H}$ since we have not demonstrated that $\mathscr{H}^{\prime}$ is the same as $\mathscr{H}$; however, the coercivity of the bilinear form allows us to assert uniqueness of solutions in the space $H_{0}^{2}(\Omega)$ as follows. Since the operator is linear, it is sufficient to show that the equation with homogeneous right side has only the zero solution. So, let $u$ be an $\mathscr{H}$-weak solution of the equation $L[u]=0$ belonging to $H_{0}^{2}(\Omega)$; then the bicontinuity of $B(\cdot, \cdot)$ on $\mathscr{H} \times H^{2}(\Omega)$ (following from (7)) implies that $B(u, u)=0$. However, since (9) must hold for $u$, this implies $\|u\|_{\mathscr{H}}=0$, and so $u \equiv 0$. This completes the proof.

Remark. Notice that the existence theorem hinges on modulus of ellipticity of the second-order part of the operator being sufficiently large so that the operator will be coercive in $H_{0}^{1}(\Omega)$, and hence in $\mathscr{H}$. All of our theorems depend on a similar condition. This does not require that the operator show explicit dependence on a parameter $\gamma$, or that the dependence, should it exist, be linear, or restricted to the $a^{i j}$ term of the operator. We choose the form given primarily because it clarifies the proofs, particularly in the regularity section that follows.

We now proceed to our uniqueness result. In the case of second-order ellipticparabolic equations, the major uniqueness results revolve around the maximum principles (strong and weak) of Fichera and Oleinik. Realizing the limitations of maximum principles for higher-order equations, we will use the Lax-Milgram Lemma, but this requires some restrictions on the third-order coefficients of the bilinear form, the $B^{i j r}$, s. The source of the restrictions is the second derivatives in the bilinear form arising from the third-order part of the operator. Where the characteristic form degenerates we must restrict the coefficients of the thirdorder part so that they do not introduce into the bilinear form derivatives that cannot be bounded by the characteristic form of the operator (and thence by the $\mathscr{H}$ norm). The desired result is, naturally, to show that the bilinear form is bicontinuous in $\mathscr{H}$, so that the conditions of Lax-Milgram hold. The condition we will use is $(\mathrm{U})$, given next.

Condition (U). There exists some constant $K$ such that at every point $x \in \bar{\Omega}$,

$$
\left[\sum_{r=1}^{n}\left(B^{i j r}(x)\right)^{2}\right]\left(\eta^{i j}\right)^{2} \leq K A^{i j r s}(x) \eta^{i j} \eta^{r s} \quad \forall \boldsymbol{\eta} n \times n \text { symmetric matrices. }
$$


Theorem 2. Let $L$ be an operator of the form given in (1) satisfying Condition (U). Then the boundary value problem (1), (4) has a unique $\mathscr{H}$-weak solution, which depends continuously on the data provided that $\gamma$ is sufficiently large.

Proof. With Condition (U), it is easy to derive from (6) that $B(u, v) \leq$ $\|u\|_{\mathscr{H}}\|v\|_{\mathscr{H}} \forall u \in \mathscr{H}$ and $v \in C_{0}^{\infty}(\Omega)$, and thus $B(\cdot, \cdot)$ can be extended in a well-defined fashion to a bicontinuous bilinear form on $\mathscr{H} \times \mathscr{H}$. Having shown above that the form is coercive for sufficiently large $\gamma$ on the dense set $C_{0}^{\infty}(\Omega)$, it is straightforward to verify the coercivity also extends to all of $\mathscr{H}$ now that the bicontinuity is established. Applying the Lax-Milgram Lemma now gives the result.

\section{REGULARITY OF SOLUTIONS}

In this section we prove the global regularity of solutions to the boundary value problem (1), (4) whose existence and uniqueness are described above. It is interesting to note that the regularity results do not depend on the uniqueness of solutions. Those readers familiar with the proof of global regularity for uniformly elliptic operators are aware that the most delicate estimates are those bounding the normal derivatives at the boundary. Our assumption is tantamount to requiring the equation to remain elliptic with respect to those normal derivatives, and so greatly facilitates the proofs of their estimates (contrasted with the results of [22], for instance, where this assumption is dropped). Our method of proof relies on the technique of elliptic regularization, the same technique used by Kohn and Nirenberg to obtain their results in [11] and [12].

To obtain the bounds in the interior, we begin by multiplying the derivatives of the solutions to the elliptically regularized boundary value problem by a cutoff function whose support is contained within $\Omega$, the domain of interest. A bound for the $H^{1}(\Omega)$ of this product function that is independent of the modulus of ellipticity of the regularized equation (denoted throughout this paper as $\varepsilon$ ) is then found. At first, this bound might appear to be worthless, since it bounds the $H^{1}$ norm of a derivative of order $\mu$ in terms of derivatives of $\mu+1$ and lower orders. What makes the bound given here useful is that the coefficient of the highest-order derivatives on the right-hand (bounding) side varies inversely with $\gamma$. Thus, by choosing $\gamma$ large enough, this term can be made arbitrarily small. It is this condition that drives the proof of the subsequent regularity theorem.

For the tangential derivatives, we introduce a cutoff function whose support has an intersection with $\partial \Omega$ sufficiently small so that the boundary can be transformed locally into a hyperplane. Derivatives in local coordinates that have no normal component are then multiplied by the new cutoff function, and the same series of estimates used to obtain the bounds in the interior of $\Omega$ are easily adapted to treat the tangential derivatives; both cases are proved simultaneously. 
We next treat the estimates necessary for the derivatives with a normal component at the boundary that are similar to those of a uniformly elliptic operator, and finally provide the general regularity result.

The elliptically regularized version of the boundary value problem is

$$
\begin{gathered}
L_{\varepsilon}[u]:=\varepsilon \Delta \Delta u+L[u]=f \quad \text { in } \Omega, \\
u=0, \quad \frac{\partial u}{\partial n}=0 \text { on } \partial \Omega,
\end{gathered}
$$

and its weak formulation requires that the solution $u \in H_{0}^{2}(\Omega)$ satisfy

$$
B_{\varepsilon}(u, v):=\varepsilon(\Delta u, \Delta v)+B(u, v)=(f, v) \quad \forall v \in C_{0}^{\infty}(\Omega)
$$

We will denote the (weak) solution to this problem by $u_{\varepsilon}$. Its existence, uniqueness, and regularity are well documented since the operator is uniformly elliptic; see, for example, Friedman [7]. Thus, we are assured that $u_{\varepsilon}$ exists as a unique weak solution, and in the lemmas below we use the regularity of the solution to obtain estimates independent of $\varepsilon$. In the concluding theorem we demonstrate that these estimates apply to the solution of the degenerate problem obtained in the limit as $\varepsilon \rightarrow 0$. The lemma providing bounds on derivatives in the interior is

Lemma 1. Assume all coefficients of $L$ belong to $C^{\mu+1}(\bar{\Omega}), u \in H^{2 \mu+2}(\Omega)$, and $\gamma>0$. Let $\varphi, \psi \in C_{0}^{\infty}(\Omega)$ with $\psi \geq 1$ on the support of $\varphi$, and $\alpha$ a multiindex of order $\mu$. Then, for $\varepsilon>0$ in a bounded set, there exist constants $C$ and $K$, independent of $\varepsilon$ and $u$, with $C$ depending on $\psi, \varphi$ and its derivatives up to order 2, the $A^{i j r s}$ and their derivatives up to order 2, and the $B^{i j r}$ and their first derivatives and $K$ depending on $\psi, \varphi$ and all coefficients and their derivatives up to order $\mu+1$, and on $\gamma$, such that the following estimate holds:

$$
\begin{aligned}
& \left|B_{\varepsilon}\left(\varphi D^{\alpha} u, \varphi D^{\alpha} u\right)-(-1)^{\mu} B_{\varepsilon}\left(u, D^{\alpha}\left[\varphi^{2} D^{\alpha} u\right]\right)\right| \\
& \quad \leq C \sum_{|\beta|=\mu+1}\left\|\psi D^{\beta} u\right\|_{0}^{2}+K \sum_{0 \leq|\beta| \leq \mu}\left\|\psi D^{\beta} u\right\|_{0}^{2} .
\end{aligned}
$$

In the second lemma we consider derivatives in directions tangent to the boundary in $\Omega \cap \Omega_{l}$ where $\Omega_{l}$ is a bounded domain that contains a portion of $\partial \Omega$ on which a suitable change of variables can be performed, as described in [13] or [21, Chapter 2.1]. In particular, in the new coordinates, which we will represent with $y$, the portion of the boundary contained in $\Omega_{l}$ is mapped into a hyperplane perpendicular to the $y_{n}$ axis. We obtain a bound on the derivatives in tangential directions within $\Omega_{l}$ as described below.

Lemma 2. Assume the coefficients, $u, \gamma, \varphi, \psi$, and $\alpha$ are as above, but with $\alpha$ having no normal component in $\Omega_{l}$ (i.e., in the changed $(y)$ coordinates, $\left.\alpha_{n}=0\right)$, and with $\varphi, \psi \in C_{0}^{\infty}\left(\Omega_{l}\right)$. Assume that $\partial \Omega \in C^{\mu+5}$. Then there exist 
constants $C$ and $K$, with the dependencies described above so that the following inequality holds:

$$
\begin{aligned}
& \left|B_{\varepsilon}\left(D^{\alpha} u, D^{\alpha} u\right)-(-1)^{\mu} B_{\varepsilon}\left(u, D^{\alpha}\left[\psi^{2} D^{\alpha} u\right]\right)\right| \\
& \quad \leq C \sum_{\substack{|\beta|=\mu+1 \\
\beta_{n} \leq 2}}\left\|\psi D^{\beta} u\right\|_{0}^{2}+K \sum_{\substack{0 \leq|\beta| \leq \mu \\
\beta_{n} \leq 2}}\left\|\psi D^{\beta} u\right\|_{0}^{2} .
\end{aligned}
$$

Here the multi-indices $\alpha$ and $\beta$ are given in $y$ coordinates, so the restriction $\beta_{n} \leq 2$ limits the number of derivatives in the normal direction. As mentioned above, the proof given establishes both lemmas.

Proof. We obtain inequalities (11) and (12) by realizing that even if we perform a smooth change of coordinates, the bilinear form can be decomposed in the following way:

$$
B_{\varepsilon}(v, w)=\sum_{m=1}^{N}\left(J_{m} D^{\lambda_{m}} v, D^{\kappa_{m}} w\right),
$$

where $J_{m}$ is a coefficient function (expressed as a function of either the $x$ or the $y$ coordinates), and $\lambda_{m}$ and $\kappa_{m}$ are multi-indices of order two or less (including order zero). Thus, we provide bounds term by term using the decomposition (13), on the difference that forms the left side of (11) and (12), and sum them to get the result. Consider the bound associated with a third-order term of the operator. The expression to be bounded can be written with the summation convention suspended as

$$
\left|\left(B_{\varepsilon}^{* i j r}\left(\varphi D^{\alpha} u\right)_{i},\left(\varphi D^{\alpha} u\right)_{j r}\right)-(-1)^{\mu}\left(B_{\varepsilon}^{* i j r} u_{i},\left(D^{\alpha}\left[\varphi^{2} D^{\alpha} u\right]\right)_{j r}\right)\right|,
$$

where it is important to note that in the case of the second lemma the coefficient $B_{\varepsilon}^{* i j r}$ is not necessarily the same as the coefficient $B^{i j r}$ derived in (5a) (because of the conversion to local coordinates all derivatives are given in $y$ coordinates and hence the coefficients of the bilinear form must be recomputed). The thirdorder coefficients in local coordinates will depend on the third- and fourth-order coefficients of the regularized operator (and hence possibly on $\varepsilon$ ), but not on lower-order coefficients (and hence not on $\gamma$ ). This explained, recall that either the support of $\varphi$ does not intersect the boundary, or $D^{\alpha}$ is purely tangential to $\partial \Omega$ within the support of $\varphi$, and hence we can apply the divergence theorem to the derivatives contained in $D^{\alpha}$ and be certain that the boundary terms will vanish. We can apply this to the second inner product in (14) to arrive at

$$
(-1)^{\mu}\left(B_{\varepsilon}^{* i j r} u_{i},\left(D^{\alpha}\left[\varphi^{2} D^{\alpha} u\right]\right)_{j r}\right)=\left(D^{\alpha}\left(B_{\varepsilon}^{* i j r} u_{i}\right),\left(\varphi^{2} D^{\alpha} u\right)_{j r}\right)
$$

(the summation convention remains suspended). Expanding both inner products in (14) using Leibniz's rule, we cancel the common term to arrive at the 
expression

$$
\begin{aligned}
\left(B_{\varepsilon}^{* i j r}\left(\varphi D^{\alpha} u\right)_{i},\left(\varphi D^{\alpha} u\right)_{j r}\right)-(-1)^{\mu}\left(B_{\varepsilon}^{* i j r} u_{i},\left(D^{\alpha} \varphi^{2} D^{\alpha} u\right)_{j r}\right) \\
=\left(\varphi_{i} B^{i j r} D^{\alpha} u,\left(\varphi D^{\alpha} u\right)_{j r}\right)-\left(\varphi \sum_{0 \leq \beta<\alpha}\left(\begin{array}{c}
\alpha \\
\beta
\end{array}\right) D^{\alpha-\beta} B_{\varepsilon}^{* i j r} D^{\beta} u_{i},\left(\varphi D^{\alpha} u\right)_{j r}\right) \\
\quad-\left(\varphi_{j} D^{\alpha}\left(B_{\varepsilon}^{* i j r} u_{i}\right),\left(\varphi D^{\alpha} u\right)_{r}\right)-\left(\varphi_{r} D^{\alpha}\left(B_{\varepsilon}^{* i j r} u_{i}\right),\left(\varphi D^{\alpha} u\right)_{j}\right) \\
\quad-\left(\varphi_{j r} D^{\alpha}\left(B_{\varepsilon}^{* i j r} u_{i}\right), \varphi D^{\alpha} u\right) .
\end{aligned}
$$

Note here that we use the notation $\beta<\alpha$ to mean that $\beta_{i} \leq \alpha_{i}$ for $1 \leq i \leq n$ with strict inequality for at least one component. Finally, we choose some derivative from $D^{\alpha}$, say, in the $s$ direction. Passing this derivative off the $u$ with $\mu+2$ derivatives in the first two terms leaves us with

$$
\begin{aligned}
& \left(\left[\varphi \varphi_{j} B_{\varepsilon}^{* i j r} D^{\alpha} u\right]_{s}, D^{\alpha-e_{s}} u_{j r}\right)-\left(\left[\varphi^{2} \sum_{0 \leq \beta<\alpha}\left(\begin{array}{c}
\alpha \\
\beta
\end{array}\right) D^{\alpha-\beta} B_{\varepsilon}^{* i j r} D^{\beta} u_{i}\right]_{s}, D^{\alpha-e_{s}} u_{j r}\right) \\
& +\left(\varphi_{i} B_{\varepsilon}^{* i j r} D^{\alpha} u-\varphi \sum_{0 \leq \beta<\alpha}\left(\begin{array}{l}
\alpha \\
\beta
\end{array}\right) D^{\alpha-\beta} B_{\varepsilon}^{* i j r} D^{\beta} u_{i}\right. \\
& \left.\varphi_{j} D^{\alpha} u_{r}+\varphi_{r} D^{\alpha} u_{j}+\varphi_{j r} D^{\alpha} u\right) \\
& -\left(\varphi_{j} D^{\alpha}\left(B_{\varepsilon}^{* i j r} u_{i}\right),\left(\varphi D^{\alpha} u\right)_{r}\right)-\left(\varphi_{r} D^{\alpha}\left(B_{\varepsilon}^{* i j r} u_{i}\right),\left(\varphi D^{\alpha} u\right)_{j}\right) \\
& -\left(\varphi_{j r} D^{\alpha}\left(B_{\varepsilon}^{* i j r} u_{i}\right), \varphi D^{\alpha} u\right)
\end{aligned}
$$

where $e_{s}$ represents the first-order multi-index in the $s$ direction. By repeatedly applying Leibniz's rule and simplifying the resulting terms using the bilinearity of the inner product, we can rewrite this expression as a sum of terms of the form $\left(J D^{\lambda} u, D^{\kappa} u\right)$, where the coefficient $J$ is a product of $B_{\varepsilon}^{* i j r}$ or its derivatives with two factors of $\varphi$ and/or its derivatives. If we now apply the $L^{2}$ Schwarz inequality and the arithmetic-geometric mean inequality to each of those terms, we arrive at a bound for the expression (14), namely,

$$
\begin{aligned}
& \left|\left(B_{\varepsilon}^{* i j r}\left(\varphi D^{\alpha} u\right)_{i},\left(\varphi D^{\alpha} u\right)_{j r}\right)-(-1)^{\mu}\left(B_{\varepsilon}^{* i j r} u_{i},\left(D^{\alpha} \varphi^{2} D^{\alpha} u\right)_{j r}\right)\right| \\
& \leq C \sum_{|\beta|=\mu+1}\left\|\psi D^{\beta} u\right\|_{0}^{2}+K \sum_{0 \leq|\beta| \leq \mu}\left\|\psi D^{\beta} u\right\|_{0}^{2} .
\end{aligned}
$$

Here we have used the fact that $\psi \geq 1$ on $\operatorname{supp} \varphi$, so $\varphi$ or any of its derivatives can be bounded by a constant times $\psi$. It is an easy matter to check that $C$ depends only on the maximum values of $B_{\varepsilon}^{* i j r}$ and its first derivatives (and not on derivatives of any higher order), while $K$ depends on derivatives of $B_{\varepsilon}^{* i j r}$ only up to order $\mu+1$. It is also easy to see that in the case of Lemma 2 , the only normal derivatives that can appear arise from the operator, i.e., only the 
$i, j$, or $r$ derivatives can be in the normal direction. Thus, considering the conversion to local coordinates, we may add the proviso that $\beta_{n} \leq 2$ to both the summation conditions in (15) in this case.

The treatment of first-order terms is analogous to the third-order term treated above, but the even-ordered terms require a trick, which we demonstrate here with a fourth-order term. Maintaining our suspension of the summation convention, applying the divergence theorem and cancelling common terms as we did above leaves us with

$$
\begin{aligned}
\left(A_{\varepsilon}^{* i j r s}\right. & \left.\left(\varphi D^{\alpha} u\right)_{i j},\left(\varphi D^{\alpha} u\right)_{r s}\right)-(-1)^{\mu}\left(A_{\varepsilon}^{* i j r s} u_{i j}, D^{\alpha}\left(\varphi^{2} D^{\alpha} u\right)_{r s}\right) \\
= & \left(A_{\varepsilon}^{* i j r s}\left(\varphi_{i} D^{\alpha} u_{j}+\varphi_{j} D^{\alpha} u_{i}+\varphi_{i j} D^{\alpha} u\right), \varphi_{r} D^{\alpha} u_{s}+\varphi_{s} D^{\alpha} u_{r}+\varphi_{r s} D^{\alpha} u\right) \\
& -\left(2 A_{\varepsilon}^{* i j r s} D^{\alpha} u_{i j}, \varphi_{r} \varphi_{s} D^{\alpha} u\right) \\
& -\left(\sum_{0 \leq \beta<\alpha}\left(\begin{array}{c}
\alpha \\
\beta
\end{array}\right) D^{\alpha-\beta} A_{\varepsilon}^{* i j r s} D^{\beta} u_{i j},\left(\varphi^{2} D^{\alpha} u\right)_{r s}\right) .
\end{aligned}
$$

The first two terms can be treated in a manner analogous to the third-order case, and are left to the reader. The last term is bounded by recognizing that in the case that $|\beta|=\mu-1$, the term is a divergence. To see this, fix $\beta$, and call $\alpha-\beta:=e_{k}$ and $D^{\beta} u:=v$. We then apply the identity

$$
v_{i j} v_{r s k}=\frac{1}{2}\left[\left(v_{i j} v_{r s}\right)_{k}+\left(v_{i k} v_{j r}\right)_{s}+\left(v_{k s} v_{i j}\right)_{r}-\left(v_{i k} v_{r s}\right)_{j}-\left(v_{k s} v_{j r}\right)_{i}\right]
$$

to get the expression

$$
\begin{aligned}
& -\left(D^{\alpha-\beta} A_{\varepsilon}^{* i j r s} D^{\beta} u_{i j},\left(\varphi^{2} D^{\alpha} u\right)_{r s}\right) \\
& =\frac{1}{2}\left\{\left(\left(\varphi^{2} D^{\alpha-\beta} A_{\varepsilon}^{* i j r s}\right)_{k} D^{\beta} u_{i j}, D^{\beta} u_{r s}\right)\right. \\
& \quad+\left(\left(\varphi^{2} D^{\alpha-\beta} A_{\varepsilon}^{* i j r s}\right)_{s} D^{\alpha} u_{i}, D^{\beta} u_{j r}\right)+\left(\left(\varphi^{2} D^{\alpha-\beta} A_{\varepsilon}^{* i j r s}\right)_{r} D^{\alpha} u_{s}, D^{\beta} u_{i j}\right) \\
& \left.\quad-\left(\left(\varphi^{2} D^{\alpha-\beta} A_{\varepsilon}^{* i j r s}\right)_{s} D^{\alpha} u_{i}, D^{\beta} u_{r s}\right)-\left(\left(\varphi^{2} D^{\alpha-\beta} A_{\varepsilon}^{* i j r s}\right)_{i} D^{\alpha} u_{s}, D^{\beta} u_{j r}\right)\right\} \\
& \quad-\left(D^{\alpha-\beta} A_{\varepsilon}^{* i j r s} D^{\beta} u_{i j},\left(\varphi^{2}\right)_{r} D^{\alpha} u_{s}+\left(\varphi^{2}\right)_{s} D^{\alpha} u_{r}+\left(\varphi^{2}\right)_{r s} D^{\alpha} u\right) .
\end{aligned}
$$

This accomplished, we proceed as we did above, applying Leibniz's rule and the Schwarz and arithmetic-geometric mean inequalities. For the case when $|\beta| \leq \mu-2$, we can simply apply the divergence theorem as we did in the third-order case to lower the order of all derivatives to $\mu+1$ or lower. This establishes the bound

$$
\begin{aligned}
& \left|\left(A^{i j r s}\left(\varphi D^{\alpha} u\right)_{i j},\left(\varphi D^{\alpha} u\right)_{r s}\right)-(-1)^{\mu}\left(A^{i j r s} u_{i j}, D^{\alpha}\left(\varphi^{2} D^{\alpha} u\right)_{r s}\right)\right| \\
& \quad \leq C \sum_{|\beta|=\mu+1}\left\|\psi D^{\beta} u\right\|_{0}^{2}+K \sum_{0 \leq|\beta| \leq \mu}\left\|\psi D^{\beta} u\right\|_{0}^{2} .
\end{aligned}
$$

This time the bounding constant $C$ depends on $A^{i j r s}$ and its derivatives up to order two, while $K$ depends on derivatives up to order $\mu+1$. It is clear that for this type of term we may introduce the restriction $\beta_{n} \leq 2$ for the Lemma 2 
case just as we did above. The lower-order terms are simpler than those treated here; we leave them for the reader. Upon summing these individual bounds, one for each term on the right side of (13), we arrive at the bounds given in (11) and (12), and this completes our proof.

We now use the previous lemmas to obtain estimates on nonnormal derivatives of solutions of the elliptically regularized problem. In the statement of the lemma we use the notation $|\cdot|_{1}$ to denote the $H^{1}$ seminorm, that is,

$$
|v|_{1}:=\left(\int_{\Omega}|\nabla v|^{2} d x\right)^{1 / 2}
$$

The constant $\tau$ mentioned in the lemma is the "second-order modulus of ellipticity" of the degenerate (and hence the regularized) operator as described in Theorem 1. That is, $\tau$ is the largest number so that

$$
\begin{array}{r}
\left\{\gamma a^{i j}(x)+C^{i j}(x)-\frac{1}{2} B_{r}^{i j r}(x)+\left[\frac{F(x)-1}{\lambda}-\left(E^{i}(x)\right)^{2}\right] \delta_{j}^{i}\right\} \xi^{i} \xi^{j} \geq \tau|\boldsymbol{\xi}|^{2} \\
\forall \boldsymbol{\xi} \in \mathbf{R}^{n} \text { and } \forall x \in \bar{\Omega} ;
\end{array}
$$

this is just an adaptation of the $\tau$ defined in inequality (8). Notice that there is no reason here that $\tau$ must be positive, although we will wish to make it so (by choice of $\gamma$ ) to obtain our regularity result.

Lemma 3. Let $u_{\varepsilon}$ be the weak solution to the homogeneous boundary value problem for the elliptically regularized equation, (10). Then assuming $f \in H^{\mu-1}(\Omega)$ and the conditions of Lemma 1, we have

(16) $\tau\left|\varphi D^{\alpha} u_{\varepsilon}\right|_{1}^{2} \leq C\left\{\left\|\psi D^{\alpha-e_{k}} f\right\|_{0}^{2}+\sum_{|\beta|=\mu+1}\left\|\psi D^{\beta} u_{\varepsilon}\right\|_{0}^{2}\right\}+K \sum_{0 \leq|\beta| \leq \mu}\left\|\psi D^{\beta} u_{\varepsilon}\right\|_{0}^{2}$

where $C$ and $K$ are independent of $\varepsilon$ and $C$ is independent of $\gamma$, with dependencies as in the first lemma. Here $e_{k}$ is a first-order multi-index whose nonzero element coincides with one in $\alpha$. Similarly, assuming $f$ is as above and the conditions of Lemma 2, we have

$$
\tau\left|\varphi D^{\alpha} u_{\varepsilon}\right|_{1}^{2} \leq C\left\{\left\|\psi D^{\alpha-e_{k}} f\right\|_{0}^{2}+\sum_{\substack{|\beta|=\mu+1 \\ \beta_{n} \leq 2}}\left\|\psi D^{\beta} u_{\varepsilon}\right\|_{0}^{2}\right\}+K \sum_{\substack{0 \leq|\beta| \leq \mu \\ \beta_{n} \leq 2}}\left\|\psi D^{\beta} u_{\varepsilon}\right\|_{0}^{2}
$$

with $C$ and $K$ as above.

Proof. First we will assume $f \in H^{2 \mu-2}(\bar{\Omega}), A^{i j r s} \in C^{2 \mu}(\bar{\Omega}), B^{i j r} \in C^{2 \mu-1}(\bar{\Omega})$, $C^{i j} \& a^{i j} \in C^{2 \mu-2}(\bar{\Omega}), E^{i} \in C^{2 \mu-3}(\bar{\Omega})$, and $F \in C^{2 \mu-4}(\bar{\Omega})$ (so that $u_{\varepsilon} \in$ $\left.H^{2 \mu+2}(\Omega)\right)$. It is easy to see from the nonnegativity of the characteristic form of the regularized operator that

$$
\tau\left|\varphi D^{\alpha} u_{\varepsilon}\right|_{1}^{2} \leq B_{\varepsilon}\left(\varphi D^{\alpha} u_{\varepsilon}, \varphi D^{\alpha} u_{\varepsilon}\right) \leq\left|(-1)^{\mu} B_{\varepsilon}\left(u_{\varepsilon}, D^{\alpha} \varphi^{2} D^{\alpha} u_{\varepsilon}\right)\right|+\mathscr{B}
$$


where $\mathscr{B}$ is the bounding (right-hand) term from (11) or (12) depending on whether $\varphi D^{\alpha}$ is an interior or tangential boundary differential operator. The last inequality is derived from the previous lemma simply by applying the triangle inequality to (11) or (12) as appropriate. Notice, however, that by the definition of the weak solution,

$$
(-1)^{\mu} B_{\varepsilon}\left(u_{\varepsilon}, D^{\alpha} \varphi^{2} D^{\alpha} u_{\varepsilon}\right)=(-1)^{\mu}\left(f, D^{\alpha} \varphi^{2} D^{\alpha} u_{\varepsilon}\right)=\left(\varphi D^{\alpha} f, \varphi D^{\alpha} u_{\varepsilon}\right)
$$

by the divergence theorem. Now we can apply the divergence theorem again to this last expression and bound as above to obtain

$$
\begin{aligned}
\left(\varphi D^{\alpha} f, \varphi D^{\alpha} u_{\varepsilon}\right) & =\left(D^{\alpha-e_{k}} f, D^{e_{k}}\left[\varphi^{2} D^{\alpha} u_{\varepsilon}\right]\right) \\
& \leq\left\|\varphi D^{\alpha-e_{k}} f\right\|_{0}^{2}+C\left\{\left\|\psi D^{\alpha} u_{\varepsilon}\right\|_{0}^{2}+\left\|\psi D^{\alpha+e_{k}} u_{\varepsilon}\right\|_{0}^{2}\right\}
\end{aligned}
$$

where $e_{k}$ is as defined above. Combining this with (18) we arrive at (16) or (17), as appropriate.

Now assume that we do not have the necessary smoothness of coefficients to apply the preceding lemmas directly to $u_{\varepsilon}$. In this case, we construct a sequence of approximating problems: homogeneous Dirichlet problems for the equations

$$
\begin{aligned}
L_{\varepsilon ; k}[u]:= & \varepsilon \Delta \Delta u+\left(A^{i j r s ; k} u_{i j}\right)_{r s}+\left(B^{i j r ; k} u_{i}\right)_{j r}-\left(C^{i j ; k} u_{i}\right)_{j} \\
& -\gamma\left(a^{i j ; k} u_{i}\right)_{j}+E^{i ; k} u_{i}+F^{; k} u=f^{; k}
\end{aligned}
$$

the coefficients of which do satisfy the smoothness requirements given at the start of the proof (as well as those of the lemma). We require $A^{i j r s ; k} \rightarrow A^{i j r s}$, $B^{i j r ; k} \rightarrow B^{i j r}, C^{i j ; k} \rightarrow C^{i j}, a^{i j ; k} \rightarrow a^{i j}, E^{i ; k} \rightarrow E^{i}$, and $F^{; k} \rightarrow F$ in $C^{\mu+1}(\bar{\Omega})$; and that $f^{; k} \rightarrow f$ in $C^{\mu-1}(\bar{\Omega})$ as $k \rightarrow \infty$. Now we can successfully apply the preceding two lemmas to the weak solutions of each of these problems to obtain (16) and (17) for $u_{\varepsilon ; k}$. But since the constants $C$ and $K$ depend only on the first $\mu+1$ derivatives of the coefficients, they converge to the corresponding constants for the coefficients of $L_{\varepsilon}$. Further, by continuous dependence on data, $u_{\varepsilon ; k}$ converges to $u_{\varepsilon}$ in $H^{\mu+3}(\Omega)$; thus, (16) and (17) will hold in the limit, that is, for $u_{\varepsilon}$ itself, and this completes our proof.

We now proceed to the estimates on normal derivatives at the boundary. The method used is somewhat tedious as derivatives with second-, third-, and fourthor higher-order normal components are each treated separately; the method of proof in each of these cases is different. The preceding lemma provided bounds on the $H^{1}$ norms of tangential derivatives at the boundary (as well as all derivatives in the interior). Thus, in fact we have bounds on derivatives with one normal component already. For this section, let $\Omega_{l}$ be an open set containing a portion of $\partial \Omega$, small enough so that a change of coordinates like that described for Lemma 2 can be performed on $\Omega_{l}$. Further, assume that $\Omega_{l}$ is chosen sufficiently small so that the characteristic form does not vanish anywhere within its closure. It follows that the coefficient of $u_{n n n n}$ in the local representation on $\Omega_{l}$ of the degenerate operator, which is the characteristic 
form in local coordinates, is bounded away from zero; say $0<\sigma \leq A^{* n n n n}$ (here, as before, the " *" is used to indicate the coefficient in local coordinates).

Lemma 4. Let the coefficients and $\partial \Omega$ be as in the lemmas above, $\Omega$ as described just above, $\gamma$ sufficiently large so that $\tau>0$, and $\chi, \psi, \varphi \in C_{0}^{\infty}(\Omega)$ with $\chi \geq 1$ on $\operatorname{supp} \psi$ and $\psi \geq 1$ on $\operatorname{supp} \varphi$. Assume that $\alpha$ is a multi-index of order $\mu-1$ with no tangential component within $\Omega_{l}$. Then there exist constants $C, C^{\prime}$, independent of $\varepsilon$ and $\gamma$, and, for every $\delta>0, K_{\delta}$ independent of $\varepsilon$ such that

$$
\begin{aligned}
\left\|\varphi D^{\alpha} u_{\varepsilon n n}\right\|_{0}^{2} \leq & \left(\delta+\frac{C}{\tau}+\frac{C^{\prime}}{\tau \delta}\right) \sum_{\substack{|\beta|=\mu+1 \\
\beta_{n} \leq 2}}\left\|\chi D^{\beta} u_{\varepsilon}\right\|_{0}^{2} \\
& +K_{\delta}\left\{\|f\|_{\mu}^{2}+\sum_{\substack{0 \leq|\beta| \leq \mu \\
\beta_{n} \leq 2}}\left\|\chi D^{\beta} u_{\varepsilon}\right\|_{0}^{2}\right\} .
\end{aligned}
$$

Proof. We begin by defining the bilinear form obtained by removing the purely normal part from $B_{\varepsilon}$. We represent the coefficients of $B_{\varepsilon}$ in local coordinates with a subscript $\varepsilon$ (to indicate their possible dependence on $\varepsilon$ ) and a superscript "*" (to indicate that a conversion to local coordinates has been made) and including $a^{i j}$ terms in $C_{\varepsilon}^{* i j}$. In the following, we sum $0 \leq i, j, r, s \leq n-1$. The definition is

$$
\begin{aligned}
B_{\varepsilon}(u, v)= & \left.A_{\varepsilon}^{* n n n n} u_{n n}, v_{n n}\right)+2\left(A_{\varepsilon}^{* n n n s} u_{n n}, v_{n s}\right)+2\left(A_{\varepsilon}^{* n n n s} u_{n s}, v_{n n}\right) \\
& +4\left(A_{\varepsilon}^{* n j n s} u_{n j}, v_{n s}\right)+\left(A_{\varepsilon}^{* n n r s} u_{n n}, v_{r s}\right)+\left(A_{\varepsilon}^{* i j n n} u_{i j}, v_{n n}\right) \\
& +2\left(A_{\varepsilon}^{* n j r s} u_{n j}, v_{r s}\right)+2\left(A_{\varepsilon}^{* i j n s} u_{i j}, v_{n s}\right)+\left(A_{\varepsilon}^{* i j r s} u_{i j}, v_{r s}\right) \\
& +\left(B_{\varepsilon}^{* n n n} u_{n}, v_{n n}\right)+2\left(B_{\varepsilon}^{* n n r} u_{n}, v_{n r}\right)+\left(B_{\varepsilon}^{* i n n} u_{i}, v_{n n}\right) \\
& +\left(B_{\varepsilon}^{* n j r} u_{n}, v_{j r}\right)+2\left(B_{\varepsilon}^{* i n r} u_{i}, v_{n r}\right)+\left(B_{\varepsilon}^{* i j r} u_{i}, v_{j r}\right) \\
& +\left(C_{\varepsilon}^{* n n} u_{n}, v_{n}\right)+\left(C_{\varepsilon}^{* n j} u_{n}, v_{j}\right)+\left(C_{\varepsilon}^{* i n} u_{i}, v_{n}\right)+\left(C_{\varepsilon}^{* i j} u_{i}, v_{j}\right) \\
& +\left(E_{\varepsilon}^{* n} u_{n}, v\right)+\left(E_{\varepsilon}^{* i} u_{i}, v\right)+\left(F^{*} u, v\right) \\
=: & \left(A_{\varepsilon}^{* n n n n} u_{n n}, v_{n n}\right)+\mathscr{T}(u, v) .
\end{aligned}
$$

By substituting $\varphi D^{\alpha} u_{\varepsilon}$ for both $u$ and $v$ in this defining equation and recalling that $A_{\varepsilon}^{* n n n n}$ is bounded below by $\sigma$, it is easy to derive

$$
\begin{aligned}
\left\|\varphi D^{\alpha} u_{\varepsilon n n}\right\|_{0}^{2} \leq & \frac{2}{\sigma}\left[B_{\varepsilon}\left(\varphi D^{\alpha} u_{\varepsilon}, \varphi D^{\alpha} u_{\varepsilon}\right)-\mathscr{T}\left(\varphi D^{\alpha} u_{\varepsilon}, \varphi D^{\alpha} u_{\varepsilon}\right)\right] \\
& +C\left[\left\|\psi D^{\alpha} u_{\varepsilon}\right\|_{0}^{2}+\left\|\psi D^{\alpha} u_{\varepsilon n}\right\|_{0}^{2}\right] .
\end{aligned}
$$

It is straightforward to apply the Schwarz inequality and the generalized arith- 
metic-geometric mean inequality to the definition of $\mathscr{T}$ to obtain the bound:

$$
\begin{aligned}
& \left|\mathscr{T}\left(\varphi D^{\alpha} u_{\varepsilon}, \varphi D^{\alpha} u_{\varepsilon}\right)\right| \leq \delta\left\|\varphi D^{\alpha} u_{\varepsilon n n}\right\|_{0}^{2} \\
& +\left(C_{1}+\frac{C_{2}}{\delta}\right)\left\{\left\|\psi D^{\alpha} u_{\varepsilon}\right\|_{0}^{2}\right. \\
& \left.\quad+\sum_{k=1}^{n}\left\|\psi D^{\alpha} u_{\varepsilon k}\right\|_{0}^{2}+\sum_{\substack{1 \leq k \leq n \\
1 \leq s \leq n-1}}\left\|\psi D^{\alpha} u_{\varepsilon k s}\right\|_{0}^{2}\right\} \\
& +K\left\|\varphi D^{\alpha} u_{\varepsilon}\right\|_{1}^{2},
\end{aligned}
$$

so it remains only to bound $B_{\varepsilon}\left(\varphi D^{\alpha} u_{\varepsilon}, \varphi D^{\alpha} u_{\varepsilon}\right)$. This is easily accomplished using Lemma 2. That is, using the rightmost inequality in (18), and (19), we arrive at

$$
\begin{aligned}
& B_{\varepsilon}\left(\varphi D^{\alpha} u_{\varepsilon}, \varphi D^{\alpha} u_{\varepsilon}\right) \\
& \quad \leq C\left\{\left\|\psi D^{\alpha-e_{k}} f\right\|_{0}^{2}+\sum_{\substack{|\beta|=\mu \\
\beta_{n} \leq 2}}\left\|\psi D^{\beta} u_{\varepsilon}\right\|_{0}^{2}\right\}+K \sum_{\substack{0 \leq|\beta| \leq \mu-1 \\
\beta_{n} \leq 2}}\left\|\psi D^{\beta} u_{\varepsilon}\right\|_{0}^{2} .
\end{aligned}
$$

In the purely normal case, i.e., when $\alpha$ is the zero multi-index, we must be more careful, but a similar bound may be obtained. Readers interested in the details are referred to [21, 33.3 , First Lemma]. Notice that all the bounding terms in (23) have order $\mu$ or less, but we wish to bound a term of order $\mu+1$. So, if we group the $C$ and $K$ terms together, calling the common coefficient $K$, and apply (22) and (23) to the appropriate terms in (21), we arrive at the following:

$$
\begin{aligned}
\left\|\varphi D^{\alpha} u_{\varepsilon n n}\right\|_{0}^{2} \leq & \delta\left\|\varphi D^{\alpha} u_{\varepsilon n n}\right\|_{0}^{2}+\left(C_{1}+\frac{C_{2}}{\delta}\right) \sum_{\substack{1 \leq k \leq n \\
1 \leq s \leq n-1}}\left\|\psi D^{\alpha} u_{\varepsilon k s}\right\|_{0}^{2} \\
& +K_{\delta}\left\{\left\|\psi D^{\alpha-e_{k}} f\right\|_{0}^{2}+\sum_{\substack{0 \leq|\beta| \leq \mu \\
\beta_{n} \leq 2}}\left\|\psi D^{\beta} u_{\varepsilon}\right\|_{0}^{2}\right\},
\end{aligned}
$$

where the $K$ mentioned earlier has been absorbed into $K_{\delta}$. We now wish to apply Lemma 3 to the $k$ and $s$ summation (with the tangential multi-index $\alpha+e_{s}$ serving as the " $\alpha$ " in (17)), but we see that the left side of (17) is an $H^{1}$ seminorm while we wish to bound the $L^{2}$ norm of a cutoff function times a derivative. This is easy to remedy using the simple estimate

$$
\|\psi \nabla v\|_{0}^{2} \leq C\left(|\psi v|_{1}^{2}+\|\chi v\|_{0}^{2}\right),
$$


(recalling the definitions of $\chi$ and $\psi$ from the statement of this lemma). Applying this with $v=D^{\alpha} u_{s}$, and applying (17) (using $\chi$ in place of $\psi$ and $\psi$ in place of $\varphi$ ) for each $1 \leq s \leq n-1$, we arrive at the following:

$$
\begin{aligned}
\left\|\varphi D^{\alpha} u_{\varepsilon n n}\right\|_{0}^{2} \leq & \left(\delta+\frac{C_{3}}{\tau}+\frac{C_{4}}{\tau \delta}\right)\left\{\left\|\chi D^{\alpha} f\right\|_{0}^{2}+\sum_{\substack{|\beta|=\mu+1 \\
\beta_{n} \leq 2}}\left\|\chi D^{\beta} u_{\varepsilon}\right\|_{0}^{2}\right\} \\
& +K_{\delta}\left\{\left\|\chi D^{\alpha-e_{k}} f\right\|_{0}^{2}+\sum_{\substack{0 \leq|\beta| \leq \mu \\
\beta_{n} \leq 2}}\left\|\chi D^{\beta} u_{\varepsilon}\right\|_{0}^{2}\right\} .
\end{aligned}
$$

Notice that we can divide inequality (17) by $\tau$ without concern by the assumption $\tau>0$. Now we simply group the $f$ terms together and bound them to arrive at (20), and this completes the proof.

In order to bound the derivatives with three normal components, we need the following well-known lemma, restated from Friedman [7].

Lemma 5. Let $v$ be an element of $L^{2}\left(\Omega \cap \Omega_{l}\right)$, and assume that the $v_{j}$ exist as weak derivatives for $1 \leq j \leq n-1$. If for any $\xi \in C_{0}^{\infty}\left(\Omega \cap \Omega_{l}\right)$ we have

$$
\left|\left(v, \xi_{n n}\right)\right| \leq P\|\xi\|_{\Omega ; 1}
$$

for some $P$ independent of $\xi$, then in any $\omega_{l} \subset \bar{\omega}_{l} \subset \bar{\Omega} \cap \Omega_{l}$ it follows that

$$
\|v\|_{\omega_{l} ; 1} \leq C\left(P+\sum_{j=1}^{n-1}\left\|v_{j}\right\|_{\Omega ; 0}+\|v\|_{\Omega ; 0}\right)
$$

for some $C$ depending only on the domains.

Notice here we indicate the norm of $H^{k}(\omega)$ by $\|\cdot\|_{\omega ; k}$

Proof. The proof for $\Omega \cap \Omega_{l}$ an $n$-dimensional hemisphere is given, for example, in Friedman's book. The adaptation for open domains is straightforward. Friedman's proof relies on the hemispherical nature of the domain only insofar as its symmetry is needed to construct a smooth extension of $v$ into the exterior of $\Omega$. Any domain $\Omega_{l}$ that is symmetric about the hyperplane $y_{n}=0$ will suffice for this purpose, and we can assume without loss of generality that our domain has this symmetry.

The necessary bound on derivatives with third-order normal components can now be obtained.

Lemma 6. Let the coefficients, $\partial \Omega, \Omega_{l}, \tau$, and $\chi, \psi, \varphi$ be as in Lemma 4 . Assume that $\alpha$ is a multi-index of order $\mu-2$ with no tangential component 
within $\Omega_{l}$. Then there exist constants $C, C^{\prime}$, independent of $\varepsilon$ and $\gamma$, and, for every $\delta>0, K_{\delta}$ independent of $\varepsilon$ such that

$$
\begin{aligned}
\left\|\varphi D^{\alpha} u_{\varepsilon n n n}\right\|_{0}^{2} \leq & \left(\delta+\frac{C}{\tau}+\frac{C^{\prime}}{\tau \delta}\right) \sum_{\substack{|\beta|=\mu+1 \\
\beta_{n} \leq 2}}\left\|\chi D^{\beta} u\right\|_{0}^{2} \\
& +K_{\delta}\left(\|f\|_{\mu-1}^{2}+\sum_{\substack{0 \leq|\beta| \leq \mu \\
\beta_{n} \leq 2}}\left\|\chi D^{\beta} u_{\varepsilon}\right\|_{0}^{2}\right) .
\end{aligned}
$$

Proof. By the reasoning leading to equation (21), we know that

$$
\left|\left(A_{\varepsilon}^{* n n n n}\left[\varphi D^{\alpha} u_{\varepsilon}\right]_{n n}, \xi_{n n}\right)\right| \leq\left|B_{\varepsilon}\left(\varphi D^{\alpha} u_{\varepsilon}, \xi\right)\right|+\left|\mathscr{T}\left(\varphi D^{\alpha} u_{\varepsilon}, \xi\right)\right|,
$$

where $\mathscr{T}$ is as defined in Lemma 4 . To derive a bound for the $\mathscr{T}$ expression here, we use the same method that gave the bound (22); we have

$$
\begin{gathered}
\left|\mathscr{T}\left(\varphi D^{\alpha} u_{\varepsilon}, \xi\right)\right| \leq C\left\{\left\|\psi D^{\alpha} u_{\varepsilon n}\right\|_{0}+\sum_{1 \leq k, l \leq n}\left\|\psi D^{\alpha} u_{\varepsilon k l}\right\|_{0}\right. \\
\left.+\sum_{\substack{1 \leq k, l \leq n \\
1 \leq i \leq n-1}}\left\|\psi D^{\alpha} u_{\varepsilon i k l}\right\|_{0}\right\}\|\xi\|_{1}+K\left\|\varphi D^{\alpha} u_{\varepsilon}\right\|_{1}\|\xi\|_{1} .
\end{gathered}
$$

To bound the $B_{\varepsilon}$ term in (28), we observe that the following decomposition holds:

$$
\begin{aligned}
& B_{\varepsilon}\left(\varphi D^{\alpha} u_{\varepsilon}, \xi\right)=\left(\varphi D^{\alpha} f, \xi\right)+\left(A_{\varepsilon}^{* i j r s} \varphi_{i} D^{\alpha} u_{\varepsilon j}, \xi_{r s}\right)+\left(A_{\varepsilon}^{* i j r s} \varphi_{j} D^{\alpha} u_{\varepsilon i}, \xi_{r s}\right) \\
& +\left(A_{\varepsilon}^{* i j r s} \varphi_{i j} D^{\alpha} u_{\varepsilon}, \xi_{r s}\right)+\left(B_{\varepsilon}^{* i j r} \varphi_{i} D^{\alpha} u_{\varepsilon}, \xi_{j r}\right) \\
& +\left(C_{\varepsilon}^{* i j} \varphi_{i} u_{\varepsilon}, \xi_{j}\right)+\left(E_{\varepsilon}^{* i} \varphi_{i} u_{\varepsilon}, \xi\right) \\
& -\sum_{0 \leq \beta<\alpha}\left(\begin{array}{c}
\alpha \\
\beta
\end{array}\right)\left[\left(\varphi D^{\alpha-\beta} A_{\varepsilon}^{* i j r s} D^{\beta} u_{\varepsilon i j}, \xi_{r s}\right)\right. \\
& +\left(\varphi D^{\alpha-\beta} B_{\varepsilon}^{* i j r} D^{\beta} u_{\varepsilon i}, \xi_{j r}\right)+\left(\varphi D^{\alpha-\beta} C_{\varepsilon}^{* i j} D^{\beta} u_{\varepsilon i}, \xi_{j}\right) \\
& \left.\quad+\left(\varphi D^{\alpha-\beta} E_{\varepsilon}^{* i} D^{\beta} u_{\varepsilon i}, \xi\right)+\left(\varphi D^{\alpha-\beta} F^{*} D^{\beta} u_{\varepsilon}, \xi\right)\right],
\end{aligned}
$$

and it is simple to derive from this that the following bound applies:

$$
\left|B_{\varepsilon}\left(\varphi D^{\alpha} u_{\varepsilon}, \xi\right)\right| \leq K\left\{\left\|\varphi D^{\alpha} f\right\|_{0}+\sum_{\substack{0 \leq|\beta| \leq \mu \\ \beta_{n} \leq 2}}\left\|\psi D^{\beta} u_{\varepsilon}\right\|_{0}\right\}\|\xi\|_{1} .
$$


Combining this with (28) and (29), we have that

$$
\begin{aligned}
& \left|\left(A_{\varepsilon}^{* n n n n}\left[\varphi D^{\alpha} u_{\varepsilon}\right]_{n n}, \xi_{n n}\right)\right| \leq\left\{C \sum_{\substack{1 \leq k, l \leq n \\
1 \leq i \leq n-1}}\left\|\psi D^{\alpha} u_{\varepsilon i k l}\right\|_{0}\right. \\
& \left.+K\left(\left\|\varphi D^{\alpha} f\right\|_{0}+\sum_{\substack{0 \leq|\beta| \leq \mu \\
\beta_{n} \leq 2}}\left\|\psi D^{\beta} u_{\varepsilon}\right\|_{0}\right)\right\}\|\xi\|_{1} .
\end{aligned}
$$

Note that this estimate is of the form (25) where $v=A_{\varepsilon}^{* n n n n}\left[\varphi D^{\alpha} u_{\varepsilon}\right]_{n n}$. Thus we can apply inequality (26) from Lemma 5 on some domain $\omega_{l}$ satisfying the conditions of the lemma and containing $\operatorname{supp} \psi$ to arrive at

$$
\begin{aligned}
\left\|A_{\varepsilon}^{* n n n n}\left[\varphi D^{\alpha} u_{\varepsilon}\right]_{n n}\right\|_{1} \leq C^{\prime}\{C & \sum_{\substack{1 \leq k, l \leq n \\
1 \leq i \leq n-1}}\left\|\psi D^{\alpha} u_{\varepsilon i k l}\right\|_{0} \\
& +K\left(\left\|\varphi D^{\alpha} f\right\|_{0}+\sum_{\substack{0 \leq|\beta| \leq k \\
\beta_{n} \leq 2}}\left\|\psi D^{\beta} u_{\varepsilon}\right\|_{0}\right) \\
& \left.+\sum_{j=1}^{n-1}\left\|\left(A_{\varepsilon}^{* n n n n}\left[\varphi u_{\varepsilon}\right]_{n n}\right)_{j}\right\|_{0}+\left\|A_{\varepsilon}^{* n n n n}\left[\varphi u_{\varepsilon}\right]_{n n}\right\|_{0}\right\} .
\end{aligned}
$$

In order to use this bound to obtain our desired result, (27), we simply apply the following easily derived estimate:

$$
\begin{aligned}
\left\|\varphi D^{\alpha} u_{\varepsilon n n n}\right\|_{0}^{2} & \leq \sigma^{-2}\left\|A_{\varepsilon}^{* n n n n} \varphi D^{\alpha} u_{\varepsilon n n n}\right\|_{0}^{2} \\
& \leq \frac{2}{\sigma^{2}}\left(\left\|A_{\varepsilon}^{* n n n n} \varphi D^{\alpha} u_{\varepsilon n n}\right\|_{1}^{2}+\left\|\left(A_{\varepsilon}^{* n n n n} \varphi\right)_{n} D^{\alpha} u_{\varepsilon n n}\right\|_{0}^{2}\right) .
\end{aligned}
$$

By squaring the inequality (30) and applying the arithmetic-geometric mean inequality, we see that this bound implies

$$
\left\|\varphi D^{\alpha} u_{\varepsilon n n n}\right\|_{0}^{2} \leq C \sum_{\substack{1 \leq k, l \leq n \\ 1 \leq i \leq n-1}}\left\|\psi D^{\alpha} u_{\varepsilon i k l}\right\|_{0}^{2}+K\left(\left\|\varphi D^{\alpha} f\right\|_{0}^{2}+\sum_{\substack{0 \leq|\beta| \leq \mu \\ \beta_{n} \leq 2}}\left\|\psi D^{\beta} u_{\varepsilon}\right\|_{0}^{2}\right) \text {. }
$$

We can now apply the result of Lemma 4, (20), to each of the terms in the first summation on the right, using the $\mu-1$ order multi-index $\alpha+e_{i}$ as the multi-index specified as $\alpha$ to obtain (27) and to complete the proof of this lemma. 
Lemma 7. Let the coefficients, $\tau, \partial \Omega, \varphi, \psi$, and $\chi$, be as in the preceding lemma. If $u_{\varepsilon}$ is the solution to the regularized problem, and $\alpha$ is a multi-index of order $\mu-2$ with $\alpha_{n}=\imath$, then there exist constants $C$ and $C^{\prime}$ independent of $\varepsilon, \delta$, and $\gamma$, and for every $\delta>0$ a constant $K_{\delta}$ independent of $\varepsilon$ such that

$$
\begin{aligned}
\left\|\varphi D^{\alpha} u_{\varepsilon n n n n}\right\|_{0}^{2} \leq & \left(\delta+\frac{C}{\tau}+\frac{C^{\prime}}{\tau \delta}\right) \sum_{\substack{|\beta|=\mu+2 \\
\beta_{n} \leq l+3}}\left\|\chi D^{\beta} u_{\varepsilon}\right\|_{0}^{2} \\
& +K_{\delta}\left(\|f\|_{\mu}^{2}+\sum_{\substack{0 \leq|\beta| \leq \mu+1 \\
\beta_{n} \leq l+3}}\left\|\chi D^{\beta} u_{\varepsilon}\right\|_{0}^{2}\right) .
\end{aligned}
$$

Proof. We proceed by induction, noting that the initial step is provided by (27) for $l=-1$. So, assume that $l_{0} \geq 0$ and (28) holds for $l<l_{0}$ (or (27) if $\left.l_{0}=0\right)$. To obtain our result for $l_{0}$, we differentiate the equation as follows:

$$
\varphi D^{\alpha} f=\varphi D^{\alpha}\left(L_{\varepsilon}\left[u_{\varepsilon}\right]\right) \text {. }
$$

By rearranging the terms, taking the $L^{2}(\Omega)$ norm and applying the triangle inequality we arrive at

$$
\begin{aligned}
\left\|A_{\varepsilon}^{* n n n n} \varphi D^{\alpha} u_{\varepsilon n n n n}\right\|_{0} & \\
\leq & \left\|\varphi D^{\alpha} f\right\|_{0}+\sum_{0 \leq \beta<\alpha+2 e_{n}}\left(\begin{array}{c}
\alpha+2 e_{n} \\
\beta
\end{array}\right)\left\|\varphi D^{\alpha+2 e_{n}-\beta} A_{\varepsilon}^{* n n n n} D^{\beta} u_{\varepsilon n n}\right\|_{0} \\
& +\sum_{\substack{1 \leq k, l, p, q \leq n \\
(k, l, p, q) \neq(n, n, n, n)}}\left\|\varphi D^{\alpha}\left(A^{k l p q} u_{\varepsilon k l}\right)_{p q}\right\|_{0} \\
& +\sum_{1 \leq k, l, p \leq n}\left\|\varphi D^{\alpha}\left(B_{\varepsilon}^{* k l p} u_{\varepsilon k}\right)_{l p}\right\|_{0}+\sum_{1 \leq k, l \leq n}\left\|\varphi D^{\alpha}\left(C_{\varepsilon}^{* k l} u_{\varepsilon k}\right)_{l}\right\|_{0} \\
& +\sum_{k=1}^{n}\left\|\varphi D^{\alpha}\left(E_{\varepsilon}^{* k} u_{\varepsilon k}\right)\right\|_{0}+\left\|\varphi D^{\alpha}\left(F u_{\varepsilon}\right)\right\|_{0} .
\end{aligned}
$$

By applying the bound $A_{\varepsilon}^{* n n n n} \geq \sigma$, and the product rule to all but the first two expressions on the right, and bounding the coefficients in our usual fashion, we have the estimate

$$
\sigma\left\|\varphi D^{\alpha} u_{\varepsilon n n n n}\right\|_{0}^{2} \leq C\left\{\left\|\varphi D^{\alpha} f\right\|_{0}^{2}+\sum_{\substack{\mu<|\beta| \leq \mu+2 \\ \beta_{n} \leq I_{0}+3}}\left\|\psi D^{\beta} u_{\varepsilon}\right\|_{0}^{2}\right\}+K \sum_{\substack{0 \leq|\beta| \leq \mu \\ \beta_{n} \leq l_{0}+3}}\left\|\psi D^{\beta} u_{\varepsilon}\right\|_{0}^{2}
$$

after squaring and applying the arithmetic-geometric mean inequality. We now apply the appropriate inequality of (31) (for $l<l_{0}$ ), (27), (20), and (17) to each of the terms in the first summation to arrive at (31) for $l=l_{0}$. Thus the lemma is proved.

We now prove the main result of this section, the existence of regular solutions. 
Theorem 3. Let $L$ be an elliptic-parabolic operator as described in $\S 2$ of this article, and assume the boundary value problem (1), (4) has no characteristic boundary (i.e., (3) is satisfied). If the coefficients of the operator belong to $C^{\mu+1}(\bar{\Omega})$, $\partial \Omega \in C^{\mu+5}$, and $f \in H^{\mu-1}(\Omega)$, then for $\gamma>0$ sufficiently large there exists an $\mathscr{H}$-weak solution of the boundary value problem, $u$, belonging to $H^{\mu+1}(\Omega)$, and for such a solution, the following bound holds:

$$
\|u\|_{\mu+1}^{2} \leq K\left(\|f\|_{\mu-1}^{2}+\|u\|_{0}^{2}\right) \text {. }
$$

Remark. The uniqueness of this smooth solution follows from the uniqueness of the $\mathscr{H}$-weak solution in $H_{0}^{2}(\Omega)$ proved in Theorem 1 .

Proof. The existence of an $\mathscr{H}$-weak solution to the degenerate problem is not in question because of Theorem 1, since we can allow $\gamma$ to become as large as necessary to guarantee a solution. We will show that estimates of the form

$$
\left|u_{\varepsilon}\right|_{\mu+1}^{2} \leq K\left(\|f\|_{\mu-1}^{2}+\left\|u_{\varepsilon}\right\|_{\mu}^{2}\right)
$$

are available for the solutions of the elliptically regularized boundary value problems, with $K$ independent of $\varepsilon$, the modulus of regularization, by assuming $\gamma$ is sufficiently large. Here, $|\cdot|_{\mu+1}$ indicates the $\mu+1$ seminorm. These estimates will then be used to show that a sequence of the $u_{\varepsilon}$ 's converge to a solution of the degenerate problem in $H^{2}(\Omega)$, which will initialize a bootstrap process leading to the desired result.

First we prove the estimates on the regularized solutions. To accomplish this, we construct a finite open covering of $\bar{\Omega}, \mathscr{O}:=\left\{\Omega_{1}, \ldots, \Omega_{\rho}\right\}$ sufficiently fine so that a conversion to local coordinates can be constructed on $\Omega_{i}$ so that its intersection with $\partial \Omega$ becomes a hyperplane for each case in which this intersection is not empty. It is well known that there is a partition of unity into $C^{\infty}\left(\mathbf{R}^{n}\right)$ functions associated with any such covering, that is, for each $1 \leq i \leq \rho$ there is associated one function (call it $g_{i}^{1}$ ) with support in $\Omega_{i}$ so that

$$
\sum_{i=1}^{\rho} g_{i}^{1}(x)=1 \quad \forall x \in \bar{\Omega}
$$

(for a proof of this fact, see Friedman [7 and 8]). Now, if we call int(supp $\left.g_{i}^{1}\right)=$ : $\Omega_{i}^{\prime}$, it is clear that the set $\mathscr{O}^{\prime}:=\left\{\Omega_{1}^{\prime}, \ldots, \Omega_{\rho}^{\prime}\right\}$ also forms a finite open covering that will have its own associated partition of unity; call the $C_{0}^{\infty}\left(\Omega_{i}^{\prime}\right)$ function $g_{i}^{2}$. We can repeat this process again to obtain a third partition of unity with functions $g_{i}^{3}$, whose support is contained in the interior of the support of the corresponding $g_{i}^{2}$. We will write $\varphi^{i}$ for $g_{i}^{3}$, and note that it satisfies the conditions for the $\varphi$ of Lemma 1 if $\Omega_{i}$ is contained in $\Omega$, and for the $\varphi$ of Lemma 2 otherwise. We can find some multiple of $g_{i}^{2}$, say $\psi^{i}:=\kappa g_{i}^{2}$, so that $\psi^{i} \geq 1$ on $\operatorname{supp} \varphi^{i}$; thus $\psi^{i}$ satisfies the conditions imposed on $\psi$ in any of the preceding lemmas. Similarly, we can find $\kappa^{\prime}$ so that $\chi^{i}:=\kappa^{\prime} g_{i}^{1} \geq 1$ on 
$\operatorname{supp} \psi^{i}$ in accordance with the requirements on $\chi$ in the previous lemmas. Note also that this $\chi^{i}$ can also serve as a $\psi$ in any of the first three lemmas. We can express the seminorm of a regularized solution as

$$
\begin{aligned}
\left|u_{\varepsilon}\right|_{\mu+1}^{2} & =\sum_{|\alpha|=\mu+1} \int_{\Omega}\left|\sum_{i=1}^{\rho} \varphi^{i} D^{\alpha} u_{\varepsilon}\right|^{2} d x \\
& \leq \rho \sum_{\substack{|\alpha|=\mu+1 \\
1 \leq i \leq \rho}} \int_{\Omega}\left|\varphi^{i} D^{\alpha} u_{\varepsilon}\right|^{2} d x=\rho \sum_{\substack{|\alpha|=\mu+1 \\
1 \leq i \leq \rho}}\left\|\varphi^{i} D^{\alpha} u_{\varepsilon}\right\|_{0}^{2} .
\end{aligned}
$$

Suppose that the $\Omega_{i}$ 's are ordered so that for $1 \leq i \leq \rho_{1}, \Omega_{i}$ is contained in $\Omega$, and the remainder intersect the boundary, $\partial \Omega$. By applying bound (24) and the estimate (16) from Lemma 3 in the interior, we have

$$
\begin{aligned}
\rho \sum_{\substack{|\alpha|=\mu+1 \\
1 \leq i \leq \rho_{1}}}\left\|\varphi^{i} D^{\alpha} u_{\varepsilon}\right\|_{0}^{2} \leq & \rho \sum_{i=1}^{\rho_{1}} \frac{C}{\tau}\left\{\left\|\chi^{i} f\right\|_{\mu-1}^{2}+\sum_{|\alpha|=\mu+1}\left\|\chi^{i} D^{\alpha} u_{\varepsilon}\right\|_{0}^{2}\right\} \\
& +K \sum_{0 \leq|\beta| \leq \mu}\left\|\chi^{i} D^{\beta} u_{\varepsilon}\right\|_{0}^{2} .
\end{aligned}
$$

To bound the terms of (34) whose support intersects the boundary, we note that applying (17) and the estimates (20), (27), and (31) term by term (as appropriate, depending on the size of $\mu$ ) gives us that

$$
\begin{gathered}
\rho \sum_{\substack{|\alpha|=\mu+1 \\
\rho_{1}<i \leq \rho}}\left\|\varphi^{i} D^{\alpha} u_{\varepsilon}\right\|_{0}^{2} \leq \rho\left(\delta+\frac{C}{\tau}+\frac{C^{\prime}}{\delta \tau}\right) \sum_{\substack{|\alpha|=\mu+1 \\
\rho_{1}<i \leq \rho}}\left\|\chi^{i} D^{\alpha} u_{\varepsilon}\right\|_{0}^{2} \\
+K_{\delta}\left(\|f\|_{\mu-1}^{2}+\left\|u_{\varepsilon}\right\|_{\mu}^{2}\right) .
\end{gathered}
$$

where the $K_{\delta}$ in the last inequality has incorporated the constant $\rho$. Now by definition, $\chi^{i} \leq \kappa^{\prime}$, so $\left(\chi^{i}\right)^{2} \leq \kappa^{\prime} \chi^{i}=\kappa^{\prime 2} g_{i}^{1}$. Thus, by adjusting $K$ appropriately and defining

$$
C(\gamma, \delta)=\rho \kappa^{\prime 2} \times \max \left\{\frac{C}{\tau},\left(\delta+\frac{C}{\tau}+\frac{C^{\prime}}{\delta \tau}\right)\right\},
$$

we can write

$$
\begin{aligned}
\rho \sum_{\substack{|\alpha|=\mu+1 \\
1 \leq i \leq \rho}}\left\|\varphi^{i} D^{\alpha} u_{\varepsilon}\right\|_{0}^{2} & \leq C(\gamma, \delta) \sum_{\substack{|\alpha|=\mu+1 \\
1 \leq i \leq \rho}} \int_{\Omega} g_{i}^{1}\left|D^{\alpha} u_{\varepsilon}\right|^{2} d x+K_{\delta}\left(\|f\|_{\mu-1}^{2}+\left\|u_{\varepsilon}\right\|_{\mu}^{2}\right) \\
& =C(\gamma, \delta) \sum_{|\alpha|=\mu+1}\left\|D^{\alpha} u_{\varepsilon}\right\|_{0}^{2}+K_{\delta}\left(\|f\|_{\mu-1}^{2}+\left\|u_{\varepsilon}\right\|_{\mu}^{2}\right) .
\end{aligned}
$$

Finally, we need only note that $C(\gamma, \delta)$ can be made less than $\rho$ for all $\gamma$ sufficiently large, by appropriate choice of $\delta$. Assuming this has been done, 
we can absorb the summation on the right into the sum on the left, and divide through to get

$$
\rho \sum_{\substack{|\alpha|=\mu+1 \\ 1 \leq i \leq \rho}}\left\|\varphi^{i} D^{\alpha} u_{\varepsilon}\right\|_{0}^{2} \leq K\left(\|f\|_{\mu-1}^{2}+\left\|u_{\varepsilon}\right\|_{\mu}^{2}\right) .
$$

We have shown in (34) that the left-hand side bounds the square of the $H^{\mu+1}$ seminorm, so we have (33) for every $\varepsilon>0$ in a bounded set. From this it follows that (32) holds for $u_{\varepsilon}$.

To see that the regularized solutions must have a convergent subsequence, note that by the uniform ellipticity of the second-order part of $L$, we know that

$$
\left\|u_{\varepsilon}\right\|_{0}^{2} \leq\left\|u_{\varepsilon}\right\|_{1}^{2} \leq \frac{1}{\tau} B_{\varepsilon}\left(u_{\varepsilon}, u_{\varepsilon}\right)=\frac{1}{\tau}\left(f, u_{\varepsilon}\right) .
$$

Calling $f / \tau=: f^{*}$ we can apply the Cauchy-Schwarz inequality to the right and obtain $\left\|u_{\varepsilon}\right\|_{0} \leq\left\|f^{*}\right\|_{0}$, and at the same time we have

$$
\left\|u_{\varepsilon}\right\|_{1}^{2} \leq\left\|f^{*}\right\|_{0}\left\|u_{\varepsilon}\right\|_{0} \leq\left\|f^{*}\right\|_{0}^{2} .
$$

Thus, by applying (32) for $\mu=1$, we have that $\left\|u_{\varepsilon}\right\|_{2}$ has a bound independent of $\varepsilon$. So, taking any sequence of $\varepsilon$ 's decreasing to zero, there must be a subsequence of the $u_{\varepsilon}$ 's weakly convergent in $H^{2}(\Omega)$, and by the Rellich compactness result, strongly convergent in $H_{0}^{1}(\Omega)$. Call the limit $u$ and it remains to show that $u$ is a solution to the degenerate problem for which (32) holds. To see that $u$ solves the problem, note that since the $H^{2}$ norm of the $u_{\varepsilon}$ 's are uniformly bounded, so are the $\left\|\Delta u_{\varepsilon}\right\|_{0}$, and so for any $v \in C_{0}^{\infty}(\Omega)$ we have

$$
B\left(u_{\varepsilon}, v\right)=(f, v)-\varepsilon\left(\Delta u_{\varepsilon}, \Delta v\right) \rightarrow(f, v) \text { as } \varepsilon \rightarrow 0 .
$$

Notice further that for $u_{\varepsilon}$ 's in our convergent sequence, weak convergence in $H^{2}$ implies $B\left(u_{\varepsilon}, v\right) \rightarrow B(u, v)$, and thus $u$ is an $\mathscr{H}$-weak solution to the degenerate problem. To see that (32) holds for $u$, we apply an induction argument. The initial step is

$$
\|u\|_{2} \leq \liminf _{\varepsilon \rightarrow 0}\left\|u_{\varepsilon}\right\|_{2} \leq \lim _{\varepsilon \rightarrow 0} K\left(\|f\|_{0}^{2}+\left\|u_{\varepsilon}\right\|_{1}^{2}\right)=K\left(\|f\|_{0}^{2}+\|u\|_{1}^{2}\right),
$$

since the $u_{\varepsilon}$ 's converge weakly to $u$ in $H^{2}$ and strongly to $u$ in $H^{1}$; this is (32) for $u$ in the case $\mu=1$. Now assume $\gamma$ is large enough so that (32) holds for $u$ in the cases $\mu<\mu_{0}$ and for the $u_{\varepsilon}$ 's in the cases $\mu \leq \mu_{0}$. Then we know that (at least a subsequence of) the $u_{\varepsilon}$ 's converge weakly in $H^{\mu_{0}+1}$ to an element as yet unknown, while they converge weakly in $H^{\mu_{0}}$ to $u$. Once again applying the Rellich compact imbedding theorem, we have that the $u_{\varepsilon}$ 's must converge strongly in $H^{\mu_{0}}$ to their weak limit in $H^{\mu_{0}+1}$, and since they already converge weakly in the larger space, the strong limit must be the same as the weak, i.e., $u$. Hence $u \in H^{\mu_{0}+1}$, and by the reasoning in (35), (32) follows for $\mu=\mu_{0}$. This completes the proof. 


\section{REFERENCES}

1. W. Bertiger and C. Cosner, Systems of second order equations with nonnegative characteristic form, Comm. Partial Differential Equations (7) 4 (1979), 701-737.

2. P. E. Chen, Bending and buckling of anisotropic plates, Composite Materials Workshop, Progress in Materials Science, Vol. I, Technomic, Stamford, Conn., 1968, pp. 217-232.

3. G. Duvaut and J.-L. Lions, Inequalities of mechanics and physics, Springer, Berlin and New York, 1976.

4. V. Esposito, Some fourth order degenerate elliptic-parabolic equations, Rend. Accad. Sci. Fis. Mat. Napoli (4) 51 (1984), 117-128.

5. G. Fichera, Sulle equazioni differenziali lineari ellitico-paraboliche del secondo ordine, Mem. Acc. Naz. Lincei, Sci. Fis. Mat. Nat. (8) 5 (1956), 1-30.

6. __ On a unified theory of boundary value problems for elliptic-parabolic equations of the second order, Proc. Sympos. on Boundary Problems in Differential Equations, R. E. Langer, Ed., Univ. of Wisconsin Press, Madison, 1960, 97-120.

7. A. Friedman, Partial differential equations, Kreiger, Malabar, Fla., 1976.

8. __ Partial differential equations of parabolic type, Prentice-Hall, Englewood Cliffs, N. J., 1964.

9. S. Goldberg, Unbounded linear operators: theory and applications, McGraw-Hill, New York, 1966.

10. W. M. Greenlee, Degeneration of a compound plate system to a membrane-plate system: $A$ singularly perturbed transmission problem, Ann. Mat. Pura Appl. (4) 128 (1981), 153-167.

11. J. J. Kohn and L. Nirenberg, Noncoercive boundary value problems, Comm. Pure Appl. Math. 18 (1965), 443-492.

12. __, Degenerate elliptic-parabolic equations of second order, Comm. Pure Appl. Math. 20 (1967), 797-872.

13. A. Kufner, O. John, and S. Fucik, Function spaces, Noordhoff, Leyden, 1977.

14. O. A. Oleinik, A boundary value problem for linear elliptic-parabolic equations, Lecture Series, 46, Univ. of Maryland Inst. Fluid Dynamics and Appl. Math., College Park, 1965.

15. __ Alcuni resultati sulle equazioni lineari e quasi lineari ellitico-paraboliche a derivate parziali del secondo ordine, Atti Accad. Naz. Lincei. Rend. Cl. Sci. Fis. Mat. Natur. (8) 40 (1966), 775-784.

16. _ _ A problem of Fichera, Soviet Math. Dokl. 5 (1964), 1129-1133.

17. $\ldots$, On the smoothness of solutions of degenerate elliptic and parabolic equations, Soviet Math. Dokl. 6 (1965), 972-975.

18. O. A. Oleinik and E. V. Radkevic, Second order equations with nonnegative characteristic form, Plenum, London, 1973.

19. R. S. Philips and L. Sarason, Singular symmetric positive first order differential operators, J. Math. Mech. 15 (1966), 235-271.

20. __ Elliptic-parabolic equations of the second order, J. Math. Mech. 17 (1968), 891-917.

21. R. G. Root, Boundary value problems for degenerate elliptic-parabolic fourth order equations, Doctoral Dissertation, Univ. of Delaware, 1988.

22. __ Self-adjoint boundary value problems for elliptic-parabolic operators of the fourth order in regions with degeneracy at the boundary (in preparation).

23. D. Tartakoff, On the regularity of non-unique solutions of degenerate elliptic-parabolic systems of partial differential equations, Comm. Pure Appl. Math. 24, (1971), 763-788.

24. S. Timoshenko and S. Woinowsky-Krieger, Theory of plates and shells, McGraw-Hill, New York, 1959.

25. R. J. Weinacht, Asymptotic distribution of eigenvalues for a class of degenerate elliptic operators of the fourth order, Rend. Mat. (7) 6 (1986), no. 1-2, 159-170 (1988). 
26. __ Degenerate elliptic equations and spongy elastic plates, Methoden und Verfahren der mathematischen Physik, Bd. 33, Problems of Applied Analysis, Peter Lang, Frankfurt, 1987, pp. 59-74.

27. __ Regularity for elliptic-parabolic operators of the fourth order (in preparation).

Department of Mathematics, Georgetown University, Washington, D. C. 20057

Current address: Department of Mathematics, Vassar College, Poughkeepsie, New York 12601 